
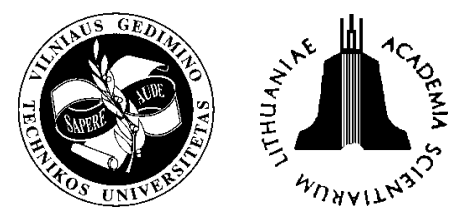

ISSN 1648-4142 print/ ISSN 1648-3480 online TRANSPORT

www.transport.vtu.lt

\title{
DETERMINATION OF EXTREME TRAIN RUNNING PARAMETERS ALONG A RAILWAY LINE SEGMENT
}

\author{
Jonas Jonaitis \\ Department of Transport Management, Vilnius Gediminas Technical University, Plytinès g. 27-407, \\ LT-10105 Vilnius, Lithuania, E-mail: Jonas.Jonaitis@ti.vtu.lt
}

Received 10 December 2005, accepted 28 March 2006

\begin{abstract}
It is possible to select train-car draft mass and traction vehicle parameters for technical speed $v_{t}$ along segment $s_{k}$ in such a way that unitary total energy consumption related to train-car mass would be the lowest. Such running is called extreme running while traction vehicle parameters and train-car mass are called optimal from the point of view of energy consumption. The optimization of traction vehicle parameters is possible if a mathematical traction vehicle model is added to the running program and then vehicle parameters are made variable and added to motion parameters as subsequent decision variables within train running program. Together with the train motion parameters optimization traction vehicle parameters should be optimized according to the criterion of unitary total energy consumption related to train-car mass. Traction vehicle optimal parameters depend on train technical speed, length and profile of segment, as well as, on train-car mass.
\end{abstract}

Keywords. train running optimization, train-car, parameters, energy consumption.

\section{Introduction}

Proper selection of train draft for a given specific transportation task is very important for an exploitation process. A train-car draft is selected for a specific traction vehicle or a traction vehicle is selected for a given train-car draft. In both cases the aim is to reduce exploitation costs down to minimum i.e.: to make traffic capacity of the line the highest and energy consumption the lowest. The line traffic capacity increases with a rise of train technical speed but at the same time energy consumption increases, so, those two parameters are contradictory. In such case it is necessary to take a middle course i.e.: for given technical speed such a train draft should be selected that unitary total energy consumption would be minimum. This question is discussed in the paper. The methods that have been used until now consisted most often of the selection of a traction vehicle for a given train-car draft; then with the use of theoretical train passage it was checked whether all the train motion conditions within a given transportation task were satisfied. The conditions are as follows [1-5]:

- $\quad$ performance of the assumed train technical speed and train maximum speed along segment $s_{k}$,

- $\quad$ performance of half maximum speed at the maximum line upward grade,
- $\quad$ traction force at the maximum speed exceeding the train motion resistance at the maximum speed by some constant.

The above described methods of traction vehicle selection for a given transportation task are approximate methods. By these methods a traction vehicle for the dynamic process of a train passage is selected in a static way. In such case, the selection of vehicle optimal parameters is not possible. Modern methods should consist of the selection of optimal train-car draft mass and traction vehicle parameters in such a way that the unitary total energy consumption related to train-car mass for given motion parameters is minimum. The task is so complicated that in the initial phase train running must be analyzed in order to examine what is the influence of train-car draft mass, traction vehicle parameters, segment length and of train technical speed on energy consumption.

At the first stage a train running along segment $s_{k}$ depending on four-axle train-car draft mass for various technical speed $v_{t}$ was analyzed. It appears that the examination of the influence of train-car mass on energy consumption, depending on train technical speed with constant traction vehicle parameters, is not possible. The unitary energy consumption will vary at a given train technical speed with various traction vehicle parameters. However, it is possible to select such 
traction vehicle parameters that energy consumption would be minimum. The parameters with which energy consumption is minimum are called optimal traction vehicle parameters. So, the examination of the influence of mass on the unitary energy consumption should be performed for traction vehicle optimal parameters. In the work [6], it has been shown that traction vehicle optimal parameters depend on train-car mass, technical speed and the length of segment $s_{k}$. Together with the increase of train technical speed, optimal power and traction vehicle optimal maximum speed increase while with the increase of the length of segment $s_{k}$ vehicle optimal power decreases. In connection with that a traction vehicle model in which traction vehicle parameters may be changed according to the changes of mass or to the train technical speed should be added to train-car draft. Such analysis is not possible with constant traction vehicle parameters. In order to perform the examination of the influence of train-car on energy consumption, depending on technical speed the above mentioned model was included into the train running program and the model parameters were made variable and added to motion parameters as subsequent decision variables. Together with motion parameters optimization traction vehicle parameters are to be optimized according to the energy consumption criterion. In such case the examination of train-car mass influence on energy consumption is performed for traction vehicle optimal parameters independently of the variations of technical speed, train-car mass or the length of segment $s_{k}$. At a given railway line segment $s_{k}$ for given train technical speed it is possible to select traction vehicle parameters and train-car draft mass in such a way that unitary total energy consumption is minimum. Such running is called extreme train running while traction vehicle parameters and train-car mass are called optimal ones. Extreme train running may be determined, if in a given transportation task motion parameters and traction vehicle parameters are simultaneously optimized. The determination of such running by means of indirect methods is not possible. When selecting a traction vehicle for a given transportation task, the authors most often were restricted to the determination of such a vehicle which meets the permissible conditions mentioned above $[2,3,5$, 7]. In the seventies of the last century, the optimization of train running along segment $s_{k}$ was made by means of a maximum rule where the work performed by the traction vehicle (energy consumption in the vehicle) in order to displace the train was taken as the criterion function. As a result, the optimum force (power) of the traction vehicle was revealed. Similar results were obtained when traction vehicle parameters were optimized and unitary energy consumption in the vehicle related to the train mass was taken as a criterion function. In the work [6], it was shown that in a traction vehicle the unitary energy consumption related to train mass monotonically decreases together with the increase of traction vehicle power, while the unitary total energy consumption (in the traction vehicle plus losses in the feeding system) related to the train-car mass is minimum. By means of such criterion it is possible to determine traction vehicle optimal parameters because the unitary total energy consumption related to the train-car mass has a minimum which is a global minimum.

\section{Train running optimization}

The train passage along the railway line segment has been reduced to the optimization task [8] in which the amount of unitary total energy consumed in the traction vehicle and in the feeding system and related to the train-car mass for displacement of the train with the mass $\mathrm{m}$, with technical speed $v_{t}$ and along the railway segment $s_{k}$ was taken as a criterion function [9]:

$$
j=\frac{m k_{w}}{3,6 m_{w_{k}} s_{k}} \sum_{i}^{n} \frac{1}{\eta^{i}} \int_{v_{0}^{i}}^{v^{i}} \frac{f(v)^{i} v^{i} d v}{\left(f(v)^{i}-w(v)^{i}\right)}\left[\frac{\mathrm{W} \cdot \mathrm{h}}{\mathrm{bt} \cdot \mathrm{km}}\right],
$$

where: $f(v)^{i} \in \Gamma-$ a set of permissible control operations-traction vehicle force $[\mathrm{N} / \mathrm{t}], n$ - the number of segment parts into which the railway line segment is divided, $v_{0}^{i}, v^{i}$ - initial and final speed within segment part $i[\mathrm{~m} / \mathrm{s}], k_{w}$ - train whirling mass coefficient, $m$ - train mass [t], $m_{w}$-train-car mass [t], $s_{k}$ - railway line segment length $[\mathrm{km}], w(v)^{i}$ - train motion resistance within segment part $i, \eta^{i}$ - total efficiency within segment part $i$.

Efficiency $\eta$ is equal to the product of the efficiency of traction vehicle driving system $\eta_{t}$, and of feeding system $\eta_{u}$ Efficiency $\eta_{t}$ depends on feeding system parameters and the distance from sub-station. Efficiency $\eta$ is determined within each segment part $n$ into which segment $s_{k}$ is divided.

The traction force for initial speed $v_{0}^{i}$ in segment part $i$ was determined in the vehicle model [10] from the following dependencies:

- for speed $v \leq v_{r}$ :

$$
f_{a d}=\left(\frac{7,5}{3,6 v+44} \frac{\mu_{0}}{0,331}+0,161\right) \frac{1000 g m_{t}}{m}\left[\frac{\mathrm{N}}{\mathrm{t}}\right] \text {, }
$$

- for speed $v>v_{r}$ :

$$
f=\frac{U_{s} I_{c} \eta_{t}}{v m} \frac{E(1-\check{s})}{\beta\left(C_{1} v+E \check{s}\right)}\left[\frac{\mathrm{N}}{\mathrm{t}}\right],
$$

where: $I_{c}$-motor continuous current [A], $E$-motor 
electromotoric force [V], $v$ - train speed $[\mathrm{m} / \mathrm{s}], \check{s}-$ motor magnetic circuit saturation degree, $\beta$ - motor magnetic circuit excitation degree, $m_{t}-$ traction vehicle mass [t], $k$ - the number of motors in a traction vehicle, $g$ - gravitational acceleration, $C_{1}-$ constant [10].

Railway line segment $s_{k}$ along which train running is performed is divided into $n$ segment parts with variable length [9]. The division was made according to the following criteria:

- the increase of distance within segment part $\Delta s \leq \Delta s_{m}$,

- the increase of speed within segment part $\Delta v \leq \Delta v_{m}$,

- no stoke-like change of traction force is permissible within the segment part.

Maximum increase in speed $\Delta v_{m}$ was selected in such a way that the approximation of train motion unitary resistance $w(v)$ and of traction vehicle unitary force $f(v)$ by means of a linear function is possible. It was also possible to average the motion unitary resistance caused by train running along the line curved parts and the line upward-grade parts within segment part I with the length equal to $\Delta s$. Motion unitary resistance $w(v)$ and traction vehicle unitary force $f(v)$ were approximated by means of tangents to the initial speed within the segment part. Proper selection of a maximum increase of distance and speed enables to determine - with required accuracy and in an effective way - train running along a railway line segment. This is ensured by a variable increase of distance $\Delta s$ within one calculation step. At start-up the distance increase most often makes several meters and it grows together with the train speed up to some dozens or even to some hundreds of meters; the length of the step is limited by a maximum increase of speed $\Delta v \leq \Delta v_{m}$, while at higher speed the increase of distance is limited by a maximum increase of distance $\Delta s \leq \Delta s_{m}$ or by the change of the railway line segment profile slope. The number and length of segment parts depend on a maximum increase of the following parameters: speed $\Delta v_{m}$, distance $\Delta s_{m}$ and the length of segment $s_{k}$ along which the train is running.

Then the optimization of the train running from the point of view of energy consumption was made. The following parameters were taken as decision variables: upper speed $-v_{g}$, the distance of running under current $s_{d}$, distance of braking start $-s_{h}$ and excitation $-\beta$. The train start-up is performed with a maximum force up to the upper speed, then running at characteristics with excitation $\beta$ is performed and when coordinate of distance $s_{d}$ is reached running from drifting to coordinate $s_{h}$ takes place. When distance $s_{h}$ is reached braking of the train is performed until the train is stopped. With the use of optimization, run- ning along the segment was determined univocally at minimum energy consumption. Repeatability and uniqueness of optimal running make its analysis possible. In order to make such analysis valuable, running must satisfy the main condition i.e.: it must map with the required accuracy - every real train running. It was assumed that the maximum increase of speed and the maximum increase of distance within a segment part make $\Delta v_{m}=2 \mathrm{~m} / \mathrm{s}, \Delta s_{m}=180 \mathrm{~m}$, accordingly.

\section{Determination of traction vehicle optimal parameters}

Determination of traction vehicle optimal parameters is possible, if a traction vehicle mathematical model [10] and a traction network numerical model [11] are added into the train running program. In the above mentioned model the parameters may be made variable in a continuous way so the optimization by means of a continuous procedure is possible. With every variation of any parameter the model determines new traction characteristics as well as new mass and mechanical gear of the traction vehicle.

Trials were made to determine vehicle optimal parameters by means of indirect methods; some simulation runnings were performed for traction vehicle parameters to be made variable in a discrete way. Then there were trials made to determine traction vehicle optimal parameters with the use of approximation methods. Unfortunately, approximation errors distorted the results to such a degree that at weak (flat) extremum of criterion function it was not possible to determine optimal parameters. It became possible when vehicle parameters as decision variables were introduced into the running program. The train running program selects the traction vehicle parameters in such a way that given running is performed at optimal total energy consumption along segment $s_{k}$. Below the parameters of DC traction vehicle are presented:

$P_{h}$ - hourly power of the traction vehicle $[\mathrm{kW}], v_{m}-$ vehicle maximum speed $[\mathrm{m} / \mathrm{s}], \alpha_{r}=\mathrm{I}_{r} / \mathrm{I}_{c}-$ relative start-up current, $\check{s}=\Phi_{c} / \Phi_{0}$ - motor magnetic circuit saturation degree, $\beta$ - motor magnetic circuit excitation degree,

where: $\mathrm{I}_{r}, \mathrm{I}_{c}-$ start-up current and continuous current of the motor, $\Phi_{c}-$ excitation stream at the motor continuous current, $\Phi_{0}$ - excitation stream at the full magnetic circuit saturation.

The introduction of the above mentioned traction vehicle parameters as decision variables into the running program is possible because they describe the vehicle traction force $(2,3)$ in the form of analytical dependency. Thus, it is possible to vary them in a con- 
tinuous way which is a necessary condition for continuous optimization. The introduction of additional decision variables into the task in which train running is optimized enlarges the size of the task. So, only the power and maximum speed of the traction vehicle were variable while the rest of the parameters were maintained constant. In the work [6], it was shown that together with the increase of start-up current $\alpha_{r}$, energy consumption decreases. However, high start-up current may cause exceeding heating of motor coils and thus the permissible temperature can be exceeded. In connection with that the following values were assumed: relative start-up current $\alpha_{r}=1,3$ and motor magnetic circuit saturation degree $\check{s}=0,7$. Traction vehicle maximum speed $v_{m}$ is the speed at which traction motors rotate with maximum angular velocity. Maximum angular velocity of traction motors depends on power. In connection with that vehicle maximum speed for given power may be changed only by the change of a traction vehicle mechanical gear. The change of a mechanical gear, the change of run of all characteristics as well as the change of minimum traction vehicle driving mass are the consequences of such change of the vehicle maximum speed for given power [10]. When optimising train running traction vehicle maximum speed $v_{m}$ may be introduced in the form of a parameter or in the form of a decision variable. In Fig 1 variations of maximum speed $v_{m}^{*}$, depending on train technical speed along segment $s_{k}=$ $15 \mathrm{~km}$ for train-car mass $m_{v}=600 \mathrm{t}$ and traction vehicle power $P_{h}=2000 \mathrm{~kW}$, are shown. Below maximum speed $v_{m}^{*}$ is presented in the form of approximation multinominal:

$$
v_{m}^{*}=33,09-1,1342 v_{t}+0,04911 v_{t}^{2}[\mathrm{~m} / \mathrm{s}] .
$$

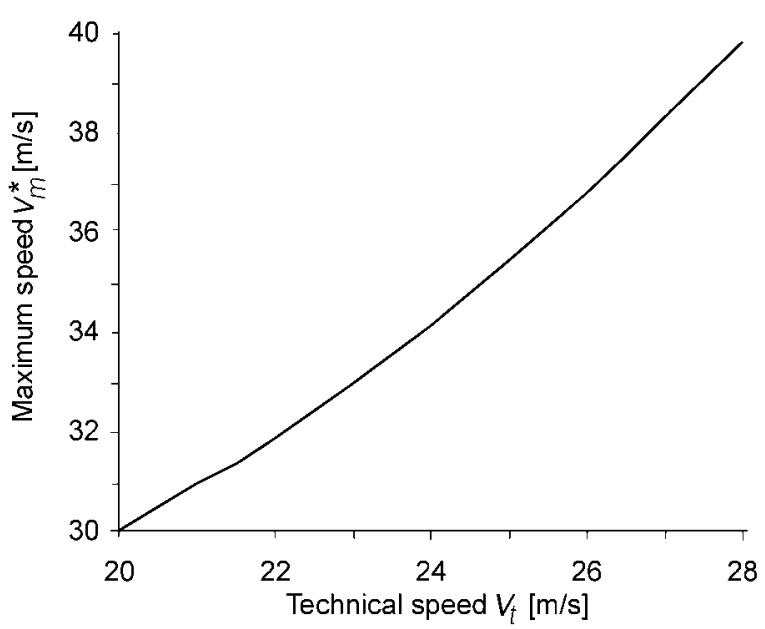

Fig 1. Variations of traction vehicle speed $v_{m}^{*}$ depending on technical speed within segment $s_{k}=15 \mathrm{~km}$
From the dependence (4), presented in Fig 1, it follows that together with increase of train technical speed traction vehicle maximum speed $v_{m}^{*}$ increases. In work [6], it was shown that technical speed $v_{m}^{*}$ depends on traction vehicle power in a small degree; in some greater degree it depends on train-car mass. Traction vehicle maximum speed $v_{m}$ has a constant value which depends on a mechanical gear, thus unitary energy consumption minimum may occur only at one speed within a given line segment. There is no doubt that future possible application of a mechanical gear enabling variation of gear ratio will bring measurable energetic advantages. It was assumed that traction vehicle maximum speed may not exceed $v_{m} \leq 50 \mathrm{~m} / \mathrm{s}$.

Then traction vehicle optimal speed was determined, depending on train technical speed. In Fig 2 variations of traction vehicle optimal power, depending on train technical speed $v_{t}$ within segment $s_{k}=$ $15 \mathrm{~km}$ for train-car mass $m_{w}=600 \mathrm{t}$, are presented. Together with train technical speed, traction vehicle optimal power increases rapidly. The increase of traction vehicle optimal power is proportional to the increase of this speed. Below traction vehicle optimal power depending on technical speed, is presented in the form of approximation multimodal:

$$
P_{h}^{*}=30,08-31,2 v_{t}+5,5155 v_{t}^{2}[\mathrm{~kW}] .
$$

In Fig 3 variations of traction vehicle optimal power, depending on the train-car mass for train technical speed $v_{t}=26 \mathrm{~m} / \mathrm{s}$, are presented. Traction vehicle optimal power increases together with the increase of train-car draft mass. Below traction vehicle optimal power depending on train-car mass for train

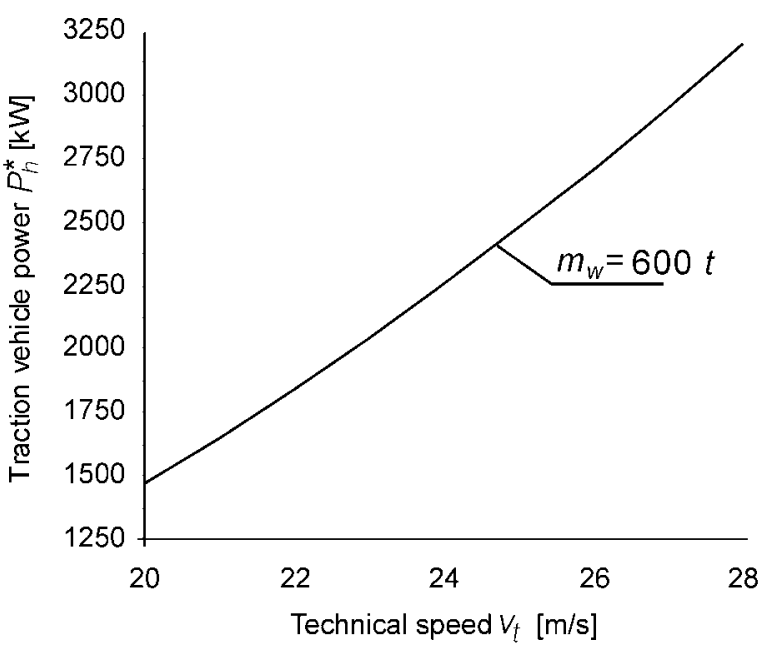

Fig 2. Variations of traction vehicle optimal power $P_{h}^{*}$ depending on technical speed within segment $s_{k}=15 \mathrm{~km}$ for train-car mass $m_{w}=600 \mathrm{t}$ 
technical speed $v_{t}=26 \mathrm{~m} / \mathrm{s}$ along segment $s_{k}=15 \mathrm{~km}$ is presented in the form of approximation multinominal:

$$
P_{h}^{*}=252,18+4,846 m_{w}-0,001267 m_{w}^{2}[\mathrm{~kW}] .
$$

From the run of the curves presented in Figures 2 and 3 , it follows that when selecting traction vehicle power for given train-car arrangement, it is necessary to take first of all the speed with which the train runs most often along the segment of the given length while in the case of various segment length, which most often occur on railway lines, when selecting traction vehicle power many factors such as line traffic capacity, transported goods mass, global consumption of energy must be taken into consideration. In such case proper selection of traction vehicle power for a given transportation task at a chosen railway line is a very complicated question. It was assumed that hourly power of BoBo- type traction vehicle is within the following range:

$$
4 \cdot 220 \leq P_{h} \leq 4 \cdot 950[\mathrm{~kW}] .
$$

\section{Determination of optimal train-car mass}

In the work [6], it was shown that train-car mass influences traction vehicle maximum speed $v_{m}^{*}$ only in a small degree, while train-car mass influences traction vehicle optimal power in a considerable degree. Together with the increase of train-car mass, also traction vehicle optimal power increases. In connection with that some tests of the influence of train-car mass on unitary energy consumption, depending on train technical speed along line segment $s_{k}=15 \mathrm{~km}$, were

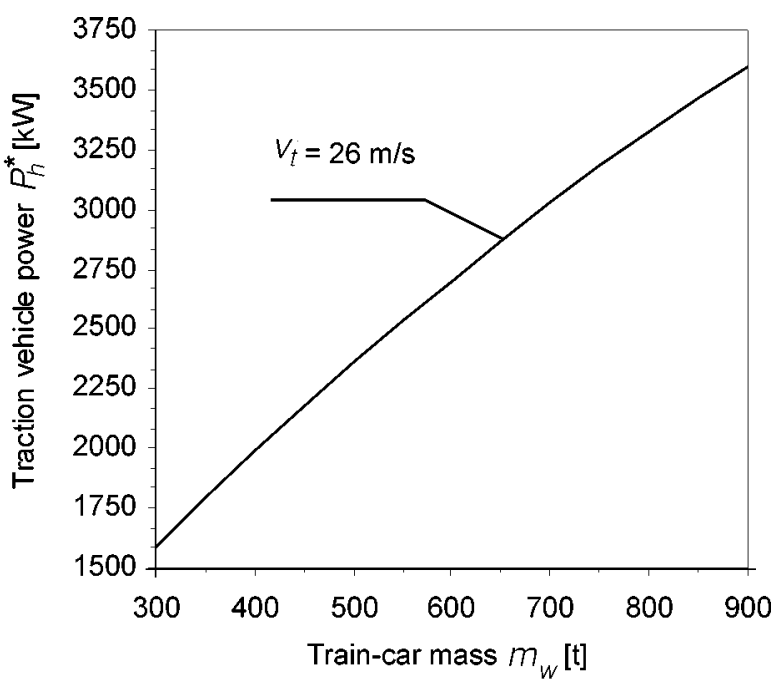

Fig 3. Variations of traction vehicle optimal power $P_{h}^{*}$ depending on train-car mass for technical speed $v_{t}=26 \mathrm{~m} / \mathrm{s}$ along segment $s_{k}=15 \mathrm{~km}$ made. Then the results of separate simulation runnings were introduced into data basis and mean-square approximation was made. Thus, some approximation multinominals of the unitary total energy consumption, related to train-car mass, were obtained. In the case of technical speed $v_{t}=26 \mathrm{~m} / \mathrm{s}$ along segment $s_{k}=15 \mathrm{~km}$, the maximum of unitary total energy consumption $(8,9)$ occurs for the train-car mass making $m_{w}=553,6 \mathrm{t}$. The increase of the train technical speed by $\Delta v=2 \mathrm{~m} / \mathrm{s}$ caused the decrease of the train-car optimal mass by about $\Delta m_{w}=97 \mathrm{t}$. The greater the mass increase the higher train technical speed is. In order to make better comparison of the diagram of the unitary energy consumption, depending on train-car mass, with the diagram of various train technical speed values, two diagrams of unitary energy consumption for the train technical speed values $v_{t}=26 \mathrm{~m} / \mathrm{s}$ and $v_{t}=$ $28 \mathrm{~m} / \mathrm{s}$ along the segment $s_{k}=15 \mathrm{~km}$ are presented in Fig 4.

Below the approximation multinominals of the unitary total energy consumption, depending on traincar mass for train technical speed $v_{t}=26 \mathrm{~m} / \mathrm{s}$ and $v_{t}=28 \mathrm{~m} / \mathrm{s}$ along segment $s_{k}=15 \mathrm{~km}$, are given:

$v_{t}=26 \mathrm{~m} / \mathrm{s}$;

$j=40,85-0,03616 m_{w}+3,266 \cdot 10^{-5} m_{w}^{2}[\mathrm{Wh} / \mathrm{bt} \cdot \mathrm{km}] ;$

$v_{t}=28 \mathrm{~m} / \mathrm{s}$;

$j=58,41-0,09503 m_{w}+9,793 \cdot 10^{-5} m_{w}[\mathrm{Wh} / \mathrm{bt} \cdot \mathrm{km}]$.

The minimum of unitary energy consumption for train technical speed $v_{t}=28 \mathrm{~m} / \mathrm{s}$ along segment $s_{k}=15 \mathrm{~km}$ occurs for train-car mass making $m_{w}=485,2 \mathrm{t}$, and together with train technical speed

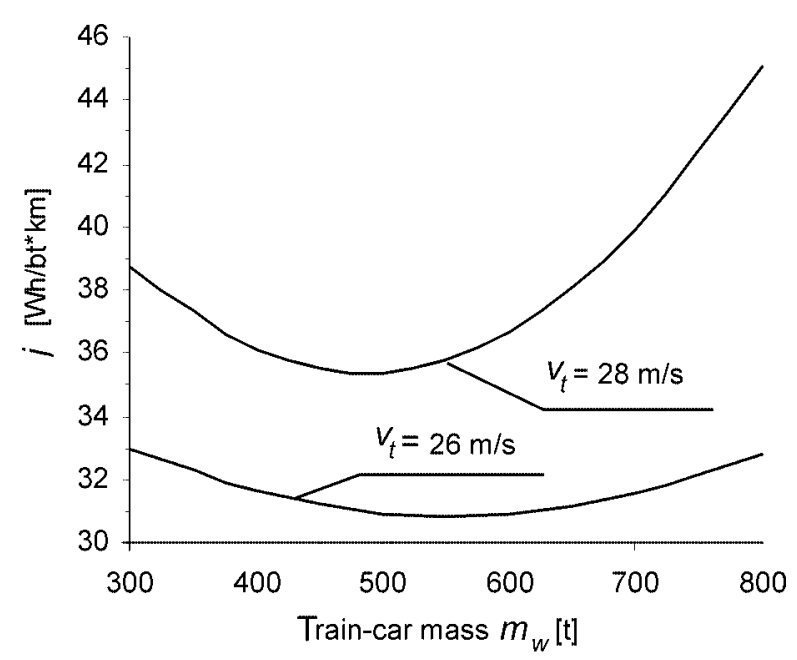

Fig 4. Variations of unitary total energy consumption depending on train mass for technical speed $v_{t}=26$ and $28 \mathrm{~m} / \mathrm{s}$ along segment $s_{k}=15 \mathrm{~km}$ 
increase the minimum is shifted to smaller train-car mass. It is easy to notice that the decrease of train-car optimal mass is not proportional to the increase of technical speed, but it varies in a non-linear way. In order to make observation of those variations better, train-car optimal mass and the increase of that mass are presented in the table below for respective train speed $v_{t}$. Train-car optimal mass is the smallest for maximum train technical speed and it increases together with a decline of that speed. The greatest mass increase occurs at intermediate technical speed and it makes $\Delta m_{w}=98,2 \mathrm{t}$ while the increase is smaller at the smallest and the greatest train technical speed and it makes $\Delta m_{w}=52,2 \mathrm{t}$ and $68,4 \mathrm{t}$, respectively.

In Fig 5 variations of train-car optimal mass $m_{w}^{*}$, depending on train technical speed along railway line segment $s_{k}=15 \mathrm{~km}$, are presented. As it follows from the curve run, the influence of train technical speed on train optimal mass is very great. Together with train technical speed train-car optimal mass monotonically decreases from the mass making $m_{w}^{*}=801 \mathrm{t}$ at speed $v_{t}=20 \mathrm{~m} / \mathrm{s}$ down to the mass making $m_{w}^{*}=485,2 \mathrm{t}$ at speed $v_{t}=28 \mathrm{~m} / \mathrm{s}$. Undoubtedly, there are more factors influencing the value of train-car optimal mass, e.g.: train-car motion resistance, length and profile of a given railway line segment etc. Two-axle train-cars have greater resistances than four-axle ones, so, we should expect that in the case of two-axle train-car drafts the optimal train-car mass will be smaller than in the case of four-axle ones. For six-axle train-car drafts the optimal train-car mass will be even less than for four-axle ones. We should suppose that railway line segment length has a greater influence on optimal mass value than the kind of train-cars.

\section{Extreme energy consumption}

It is possible to select train technical speed and then traction vehicle parameters for given train-car mass along a railway line segment with the given length in such a way that extreme energy consumption would occur. Such running is called train extreme running, while train-car mass and traction vehicle parameters are called optimal ones. When train-car mass is increased or decreased at the same train technical speed and at the same traction vehicle parameters, energy consumption increases. As it was shown in the work [6], train-car optimal mass depends on train technical speed as well as on railway line segment length see Fig 4 and 5. Together with the increase of train technical speed $v_{t}$ train-car optimal mass $m_{w}$ decreases because the traction vehicle optimal parameters depend also on motion parameters - maximum speed $m_{w}^{*}$ depends on train technical speed and power $P_{h}^{*}$ depends on technical speed, as well as, on train- car mass. Thus, extreme running of a given train draft along segments with various length is not possible. In order to illustrate this question in Table 1 and in Fig 6 the unitary energy consumption along the railway line segment with length of $s_{k}=15 \mathrm{~km}$ for various trac-

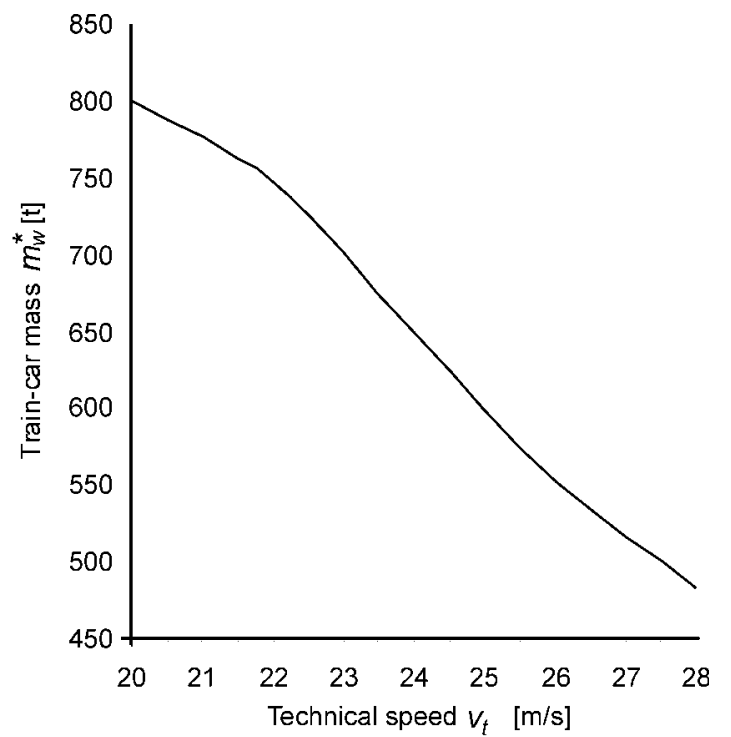

Fig 5. Variation of train-car optimal mass $m_{w}^{*}$ depending on train technical speed along segment $s_{k}=15 \mathrm{~km}$

Table 1. Variation of train-car optimal mass $m_{w}^{*}$

\begin{tabular}{|c|c|c|c|c|c|}
\hline$v_{t}[\mathrm{~m} / \mathrm{s}]$ & 20 & 22 & 24 & 26 & 28 \\
\hline$m_{w}{ }^{*}[\mathrm{t}]$ & 801 & 748,8 & 650,6 & 553,6 & 485,2 \\
\hline$\Delta m_{w}[\mathrm{t}]$ & & $-52,2$ & $-98,2$ & -97 & $-68,4$ \\
\hline
\end{tabular}

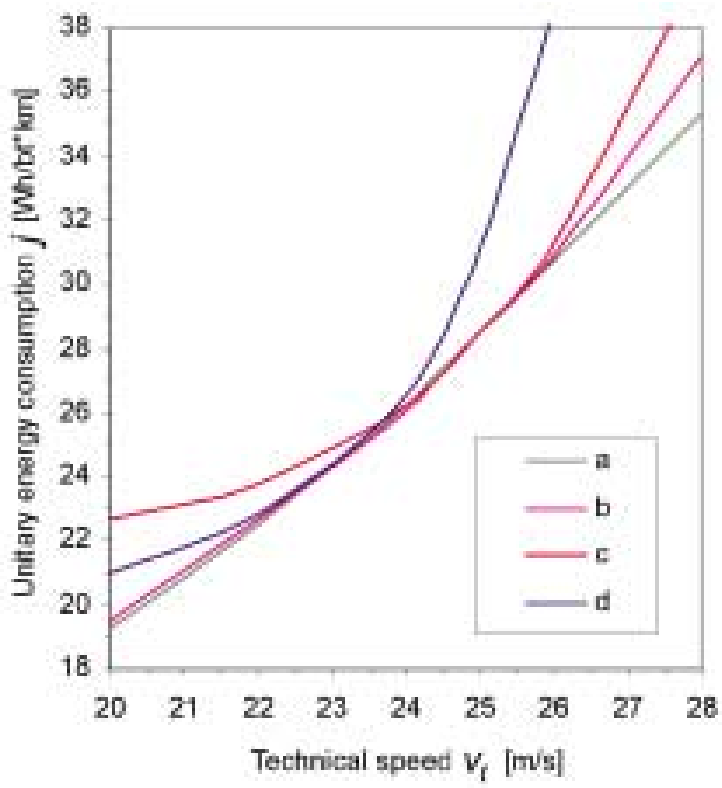

Fig 6. Variations of unitary energy consumption 
tion vehicle parameters and various train-car mass values depending on technical speed $v_{t}$ is presented:

a) $P_{h}^{*}, v_{m}^{*}, m_{w}^{*}:$

$j_{1}^{*}=35,75-3,061 v_{t}+0,1122 v_{t}^{2}-$

$3,569 \cdot 10^{-5} \exp \left(0,4 v_{t}\right)[\mathrm{Wh} / \mathrm{bt} \cdot \mathrm{km}]$;

b) $P_{h}^{*}, v_{m}^{*}, m_{w}=650 \mathrm{t}$

$j_{2}=-159,4-0,2278 v_{t}-0,376 v_{t}^{2}+$

$122,8 \exp \left(0,05 \mathrm{v}_{t}\right)[\mathrm{Wh} / \mathrm{bt} \cdot \mathrm{km}]$;

c) $P_{h}=2570 \mathrm{~kW}, v_{m}=36 \mathrm{~m} / \mathrm{s}, m_{w}=550 \mathrm{t}$

$j_{3}=-18,67+5,083 v_{t}-0,2694 v_{t}^{2}+$

$3,896 \exp (0,125 \mathrm{v} t)[\mathrm{Wh} / \mathrm{bt} \cdot \mathrm{km}]$;

d) $P_{h}=2400 \mathrm{~kW}, v_{m}=34 \mathrm{~m} / \mathrm{s}, m_{w}=650 \mathrm{t}$

$j_{4}=-18,72+3,397 v_{t}-0,07164 v_{t}^{2}+$

$1,146 \cdot 10^{-6} \exp \left(0,64 v_{t}\right)[\mathrm{Wh} / \mathrm{bt} \cdot \mathrm{km}]$.

Curve $a$ presents the geometrical point of the extreme of energy consumption depending on train technical speed along the railway line segment. The curve was traced at traction vehicle optimal parameters $P_{h}^{*}, v_{m}^{*}$, as well as, at train-car optimal mass $m_{w}^{*}$. Together with train technical speed increase, train-car optimal mass $m_{w}^{*}$ decreases while optimal power $P_{h}^{*}$ and traction vehicle maximum speed increase. So, at the given traction vehicle optimal parameters and train-car optimal mass only one point of the above mentioned curve may occur. Curve $b$ was traced at traction vehicle optimal parameters $P_{h}^{*}, v_{m}^{*}$, as well as, at train-car mass making $m_{w}^{*}=650 \mathrm{t}$. The comparison of those curve runs shows that at small train technical speed the unitary energy consumption is more or less the same. Significant differences occur at greater train technical speed values. It means that at greater train technical speed a given train draft approaches forcing speed because train-car mass is greater that the optimal mass which makes $m_{w}^{*}=$ $485,2 \mathrm{t}$ for speed $v_{t}=28 \mathrm{~m} / \mathrm{s}$ and that is the reason why energy consumption increases so rapidly. Curve $c$ is tangent to the extreme curve of energy consumption for speed $v_{t}=26 \mathrm{~m} / \mathrm{s}$ while curve $d$ is tangent to it for speed $v_{t}=24 \mathrm{~m} / \mathrm{s}$ as for those technical speed values both train-car mass and traction vehicle parameters are optimal. The data described above are included in Table 2.

In this work it is shown that traction vehicle optimal parameters depend on train technical speed $v_{t}$, train-car draft mass $m_{w}$, the length of railway line segment $s_{k}$, feeding system parameters as well as on some other factors such as train traffic intensity, railway line profile, train-car type etc. Variations of any factors mentioned above can change traction vehicle optimal parameters and train-car optimal mass.

Table 2. Variation of the unitary energy consumption

\begin{tabular}{|c|c|c|c|c|c|c|}
\hline$v_{t}[\mathrm{~m} / \mathrm{s}]$ & 20 & 22 & 24 & 26 & 28 & Curve \\
\hline$j_{1}^{*}$ & 19,27 & 22,5 & 26,3 & 30,84 & 35,36 & \\
$P_{h}^{*}[\mathrm{~kW}]$ & 1800 & 2100 & 2400 & 2570 & 2800 & \\
$v_{m}^{*}[\mathrm{~m} / \mathrm{s}]$ & 30 & 32 & 34 & 36 & 39 & $\mathrm{a}$ \\
$m_{w}^{*}[\mathrm{t}]$ & 801 & 748,8 & 650,6 & 553,6 & 485,2 & \\
\hline$j_{2}$ & 19,5 & 22,63 & 26,4 & 31,2 & 37,5 & \\
$P_{h}^{*}[\mathrm{~kW}]$ & 1500 & 1919 & 2400 & 2831 & 3353 & $\mathrm{~b}$ \\
$v_{m}^{*}[\mathrm{~m} / \mathrm{s}]$ & 30 & 32,2 & 34 & 37 & 39,6 & \\
\hline$j_{3}$ & 22,68 & 23,8 & 26,8 & 32 & 43,2 & $\mathrm{c}$ \\
\hline$j_{4}$ & 20,98 & 22,8 & 26,3 & & & $\mathrm{~d}$ \\
\hline
\end{tabular}

\section{Conclusions}

1. It has been shown that the optimization of traction vehicle parameters is possible when a mathematical model of a traction vehicle is included into a given running program and then traction vehicle parameters are made variable and added to the motion parameters as subsequent decision variables in the train running program. Together with train motion parameters optimization traction vehicle parameters are to be optimized according to the criterion of the unitary total energy consumption related to the train-car mass.

2. As result of the analysis of train running depending on traction vehicle power, vehicle maximum speed, and train-car mass, it was demonstrated that traction vehicle maximum speed $v_{m}^{*}$ only in a small degree depends on train-car mass, traction vehicle power and railway line segment along which train running takes place [6], but it depends on train technical speed. Moreover, it increases almost proportionally to that speed - see Fig 1. As traction vehicle maximum speed $v_{m}^{*}$ has a constant value which depends on mechanical gear and traction motor power, the minimum of energy consumption may occur only at one technical speed $v_{t}$ along a given railway line segment $s_{k}$.

3. It was shown that along railway line segment $s_{k}$ for technical speed $v_{t}$, it is possible to select some train-car draft mass and some traction vehicle parameters in such a way that unitary total energy consumption, related to train-car mass, would be minimum. Such running was called extreme one and traction 
vehicle parameters, as well as, train-car mass were called optimal from the point of the view of energy consumption. Traction vehicle optimal parameters depend on train technical speed, length and profile of a given railway line segment $s_{k}$, as well as, on traincar mass. However, the method presented cannot be applied to optimize traction vehicle parameters along a chosen railway line with various length of segments, because for such optimization it is necessary to use a hierarchic procedure in which decision variables of a vehicle would be placed on a higher level while decision variables of motion for particular segments would be placed on a lower level.

\section{References}

1. Frackowiak, J. Optimization of electric energy consumption by traction vehicles driven by means of series motors fed by choppers. In: Proceedings of international conference "MET" 01". Gdansk, 2001, p. 177-183.

2. Jaworski, Cz. The theory of electric traction. Warsaw: WK, 1996. 265 p.

3. Kaluza, E. The book of questions and exercises on electric traction. Gliwice: Politechnika, 1994. 288 p.

4. Mierzejiewski, L. The influence of rolling stock with energy recuperation on the conditions of DC current traction power engineering system functioning. In: Proceedings of international conference "MET" 01". Gdansk, 2001, p. 131-137.

5. Podoski, M.; Kacprzak, J. The principles of electric traction. Warsaw: WK, 1980. 323 p.

6. Wnuk, M. Train energo-optimal running along a railway section. Warsaw: PW, 1994. 33 p.

7. Jaworski, Cz. Approximation of traction motor characteristics. Warsaw, 1970. $288 \mathrm{p}$.

8. Findeisen, W.; Szymanowski, W., Wierzbicki, A. The theory and the calculation methods for optimization. Warsaw: PWN, 1980. 380 p.

9. Wnuk, M. The mathematical model of traction locomotive. Warsaw: PW, 1993. 29 p.

10. Wnuk, M. Digital model of DC current traction network. Warsaw: PW, 1993. 33 p.

11. Pospisil, M. Optimization of electric locomotives for local passage routes with $\mathrm{AC}$ feeding $-25 \mathrm{kV}, 50 \mathrm{~Hz}$. In: Proceedings of international conference "MET" 97", Warsaw, 1997, p. 131-138. 